\title{
ON THE QUADRICS ASSOCIATED WITH A POINT OF A SURFACE
}

\author{
SU-CHENG CHANG ${ }^{1}$
}

It is well known that among the three-parameter family of quadrics having a contact of the second order with an analytic surface at a point there are two systems of quadrics known as the quadrics of Darboux and the quadrics of Moutard. The particular quadric of the first family introduced in this note is of some interest. We propose to give a new geometrical construction for the cone of Segre by using Moutard quadrics and study the canonical pencil of lines associated with the surface.

Let $x^{i}(u, v)(i=1, \cdots, 4)$ be the projective normal coordinates of a point on a surface $S$ referred to its asymptotic net $(u, v)$; then the functions $x$ are solutions of a completely integrable system of differential equations in Fubini's canonical form

$$
\begin{aligned}
x_{u u} & =\theta_{u} x_{u}+\beta x_{v}+p_{11} x, \\
x_{v v} & =\gamma x_{u}+\theta_{v} x_{v}+p_{22} x, \\
\theta & =\log \beta \gamma .
\end{aligned}
$$

As usual we introduce nonhomogeneous local coordinates of a point with respect to the tetrahedron $\left(x_{u} x_{u} x_{v} x_{u v}\right)$, so that the equation of an $y$ quadric of Darboux is found to be

$$
z-x y+k z^{2}=0,
$$

where $k$ denotes a parameter, while the equation of the quadric of Moutard which belongs to the tangent $y-n x=0, z=0$ is

$$
\begin{aligned}
{\left[4\left(\beta+\gamma n^{3}\right)^{2}\right.} & \left.+3 n\left(\beta_{u}+4 \beta_{v} n+4 \gamma_{u} n^{3}+\gamma_{v} n^{4}\right)\right] z^{2} \\
+ & 36 n^{8}\left[z-x y+\frac{z^{2}}{2} \frac{\partial^{2} \log \beta \gamma}{\partial u \partial v}\right] \\
& -12 n\left(\beta-2 \gamma n^{3}\right)\left(y+\frac{1}{2} \frac{\partial \log \beta \gamma}{\partial u} z\right) z \\
& -12 n^{2}\left(\gamma n^{8}-2 \beta\right)\left(x+\frac{1}{2} \frac{\partial \log \beta \gamma}{\partial v} z\right) z=0 .
\end{aligned}
$$

Received by the editors May 22, 1944.

1 The stay of the author in the National University of Chekiang was made possible by the Board of Trustees of the Indemnity Fund remitted by the British government. 
These quadrics intersect each other in the asymptotic tangents and a conic in the plane

$$
\begin{aligned}
& z\left[4\left(\beta+\gamma n^{3}\right)^{2}+3 n\left(\beta_{u}+\right.\right.\left.4 \beta_{v} n+4 \gamma_{u} n^{3}+\gamma_{v} n^{4}\right)+36 n^{3} K \beta \gamma \\
&\left.-6 n \frac{\partial \log \beta \gamma}{\partial u}\left(\beta-2 \gamma n^{3}\right)-6 n^{2} \frac{\partial \log \beta \gamma}{\partial v}\left(\gamma n^{3}-2 \beta\right)\right] \\
& \quad-12 n\left(\beta-2 \gamma n^{3}\right) y-12 n^{2}\left(\gamma n^{3}-2 \beta\right) x=0,
\end{aligned}
$$

where we have placed

$$
k=\frac{1}{2} \frac{\partial^{2} \log \beta \gamma}{\partial u \partial v}-K \beta \gamma .
$$

The plane coordinates of this plane are evidently

$$
\begin{aligned}
u_{0}= & 0, \\
\rho u_{1}= & -12 n^{2}\left(\gamma n^{3}-2 \beta\right), \quad \rho u_{2}=-12 n\left(\beta-2 \gamma n^{3}\right), \\
\rho u_{3}= & 4 \beta^{2}-3 \beta \phi+12 \beta n^{2} \psi+(8 \beta \gamma+36 K \beta \gamma) n^{3}+12 \gamma \phi n^{4} \\
& -3 \gamma n^{5} \psi+4 \gamma^{2} n^{6},
\end{aligned}
$$

where $\phi=\partial \log \beta \gamma^{2} / \partial u$ and $\psi=\partial \log \beta^{2} \gamma / \partial v$, so that the cone enveloped by it may be obtained by eliminating $n$ from the equations

$$
\begin{aligned}
& \pi n^{3}+\omega n^{2}+t n+s=0, \\
& \sigma n^{3}+\tau n+\omega n+p=0,
\end{aligned}
$$

where

$$
\begin{aligned}
\omega & =7 u_{1} \psi / 2+7 u_{2} \phi / 2-6 u_{3}, & & \\
\pi & =u_{2} \psi-2 \gamma u_{1}, & p & =u_{1} \phi-2 \beta u_{2}, \\
s & =(4+6 K) \beta u_{1}, & \sigma & =(4+6 K) \gamma u_{2}, \\
t & =u_{1} \phi+6(1+2 K) \beta u_{2}, & \tau & =u_{2} \psi+6(1+2 K) \gamma u_{1} .
\end{aligned}
$$

The result of carrying out the computation for a given value of $K$ is

$$
\left|\begin{array}{cccc}
\pi & \omega & t & s \\
\sigma & \tau & \omega & p \\
0 & p \pi-\sigma s & \omega p-\tau s & p t-\omega s \\
\sigma \omega-\pi \tau & \sigma t-\pi \omega & \sigma s-\pi p & 0
\end{array}\right|=0 .
$$

It should be remarked that when and only when $K=-2 / 3$ the equation of the cone enveloped by the planes (5) is different from (7), 
since the latter in this case becomes an identity. From (2) and (4) we may infer naturally that in the pencil of Darboux the quadric

$$
z-x y+\left(\frac{1}{2} \frac{\partial^{2} \log \beta \gamma}{\partial u \partial v}+\frac{2}{3} \beta \gamma\right) z^{2}=0
$$

differs from other quadrics by a certain geometrical characterization.

The third polar of $z=0$ with respect to the cone (7) is

$$
6 u_{1} u_{2} u_{3}-3\left(u_{1} \psi+u_{2} \phi\right) u_{1} u_{2}-\left(\beta u_{2}^{3}+\gamma u_{1}^{3}\right)=0,
$$

which does not contain $K$. That is to say, all the cones (7) with different values of $K$ have the third polar of $z=0$ in common.

As to the second polar of the same plane $z=0$ we have

$$
[\cdots] u_{1} u_{2}+u_{1} u_{2} \omega_{2}^{2}+6\left[\left(u_{1} \psi+u_{2} \phi\right) u_{1} u_{2}-2\left(\beta u_{2}^{3}+\gamma u_{1}^{3}\right)\right]=0
$$

where $[\cdots]$ does not contain $\omega$. Hence through each tangent of the surface $S$ at $P$ there are besides $z=0$ two tangent planes $p_{1}$ and $p_{2}$ of the cone (10) and the harmonic conjugate plane of $z=0$ with respect to $p_{1}$ and $p_{2}$ just envelopes the cone (9). Thus we obtain a correspondence between a tangent of the surface and a plane through it. Especially, the three corresponding planes of the tangents of Darboux are concurrent in the first directrix of Wilczynski.

The equation of the cone (9) in point coordinates is found to be

$$
\begin{aligned}
-3\left(y+\frac{\phi}{2} z\right)^{2}\left(x+\frac{\psi}{2} z\right)^{2}+2 \gamma z\left(y+\frac{\phi}{2} z\right)^{3} \\
+2 \beta z\left(x+\frac{\psi}{2} z\right)^{3}-\frac{3}{2} \beta \gamma\left(x+\frac{\psi}{2} z\right)\left(y+\frac{\phi}{2} z\right) z^{2} \\
+\frac{\beta^{2} \gamma^{2}}{16} z^{4}=0 .
\end{aligned}
$$

It follows that $z=0$ is the only double tangent plane along the two asymptotic tangents of $S$ and therefore that there are three cuspidal generators, each passing through one of the points

$$
\left(0, \frac{\omega^{2}}{2}\left(\beta \gamma^{2}\right)^{1 / 3}-\frac{\psi}{2}, \frac{\omega}{2}\left(\gamma^{2} \beta\right)^{1 / 3}-\frac{\phi}{2}, 1\right), \quad\left(\omega^{3}=1, \omega \neq 1\right) .
$$

The harmonic conjugate line of $z=0$ with respect to the trihedral of the three cuspidal generators is also the first directrix of Wilczynski. Furthermore the three cuspidal tangent planes of the cone (9) are concurrent in the same directrix. 
It is worth noticing that the cones (10) with variable $K$ have a common tangent plane which passes through each asymptotic tangent. The two planes thus obtained intersect in the canonical line $C(-7 / 12) .^{2}$

Let us now attend to the particular quadric (8). Eliminating $n$ from the equations

$$
\begin{gathered}
\pi n^{2}+\omega n+p=0, \\
\beta u_{1}\left(1-2(\gamma / \beta) n^{3}\right)=n\left(\gamma n^{3}-2 \beta\right) u_{2},
\end{gathered}
$$

we obtain that the cone in this case is

$$
\left|\begin{array}{cccc}
p \gamma u_{2} & 2 \gamma u_{1} p & \pi \beta u_{1} & \omega \beta u_{1}-2 \beta u_{2} p \\
-2 \gamma \pi u_{1}+\gamma u_{2} \omega & \gamma u_{2} p & 2 \beta \pi u_{2} & \beta u_{1} \pi \\
\pi & \omega & p & 0 \\
0 & \pi & \omega & p
\end{array}\right|=0 .
$$

The second polar of the plane $z=0$ with respect to this cone is

$$
-u_{1} u_{2} \omega^{2}+\left(u_{1}^{2} \pi+u_{2}^{2} p\right) \omega=0 .
$$

Therefore the quadric (8) is distinguished from other quadrics in the pencil of Darboux by the fact that the corresponding cone (12) decomposes into a cone of the third class and a pencil of planes with $C(-7 / 12)$ as its axis. Let $p_{1}$ and $p_{2}$ be the two tangent planes of the cone (12) through a given tangent of $S$ at $P$ but distinct from $z=0$, and $t$ a plane through the same tangent such that

$$
\left(z=0, t ; p_{1}, p_{2}\right)=\tau=\text { const. }
$$

Then the equation of $t$ is easily found to be

$$
\begin{aligned}
(1-\tau)\left(u_{1} x+u_{2} y\right)+z\left[\frac{7}{12}\left(\psi u_{1}+\phi u_{2}\right)\right. & -5 \tau\left(\psi u_{1}+\phi u_{2}\right) \\
& \left.-\frac{\tau}{3}\left(\frac{\gamma u_{1}^{2}}{u_{2}}+\frac{\beta u_{2}^{2}}{u_{1}}\right)\right]=0,
\end{aligned}
$$

$u_{1} / u_{2}$ being a parameter. This plane envelopes a cone of the fourth order with three cuspidal tangent planes concurrent in the canonical line $C(-(7-5 K) / 12(1-K))$, while the latter is also the polar line of $z=0$ with respect to the trihedral of the three cuspidal generators.

Finally, we shall give a geometrical interpretation to the cone of

${ }^{2}$ This line appeared in Sannia's paper. See Rendiconti R. Accademia dei Lincei (6) vol. 9 (1929) pp. 1081-1085. 
Segre. Consider the two quadrics of Moutard belonging to the tanents $t$ and $t^{\prime}$ at a point of the surface; they intersect in the asymptotic tangents and a conic. The limiting position of the plane of this conic as $t^{\prime} \rightarrow t$ is

$$
\begin{aligned}
z\left[4 \beta^{2}-4 \gamma^{2} n^{6}-2 \beta n \phi+\right. & \left.2 \gamma n^{5} \psi+4 \beta \psi n^{2}-4 \gamma \phi n^{4}\right] \\
+ & 8\left(\gamma n^{8}+\beta\right) n^{2} x-8\left(\beta+\gamma n^{3}\right) n y=0,
\end{aligned}
$$

which envelopes the cone of Segre.

National University of Chekiang

\section{ON A REPRESENTATION IN SPACE OF GROUPS OF CIRCLE AND TURBINE TRANSFORMATIONS IN THE PLANE}

\section{J. M. FELD}

1. Introduction. In a previous paper [6] the author showed that the oriented lineal elements in the euclidean plane can be mapped continuously and $(1,1)$ upon the points of quasi-elliptic ${ }^{2}$ three-space $Q_{3}$ so that the whirl-similitude group of turbine ${ }^{3}$ transformations in the euclidean plane is represented isomorphically upon the group of projective automorphisms of $Q_{3}$. By means of this representation proper turbines in the plane are mapped upon those real lines in $Q_{3}$ which do not intersect a line $L$, the real part of the quasi-elliptic absolute. It is the purpose of this note to investigate this representation analytically and to extend it so as to yield a $(1,1)$ continuous mapping of the turbines (proper and improper) in the Moebius plane upon all the lines in projective $S_{3}$. By such means we establish the isomorphism between certain groups of projective transformations in space on the one hand, and on the other of Kasner's 15-parameter group of turbine transformations [7] and some of its important subgroups, namely the Moebius, Laguerre, and Lie groups of circle transformations.

Other representations of turbines in space are due to Kasner and DeCicco [7, 8] and to A. Narasinga Rao [9]. The former use a

Presented to the Society, February 26, 1944; received by the editors April 17, 1944.

1 The numbers in brackets refer to the bibliography at the end of this paper.

2 The term quasi-elliptic space is due to Blaschke $[1,2]$. The absolute of this space is composed of a pair of conjugate imaginary planes $x_{3}^{2}+x_{4}^{2}=0$ and a pair of conjugate imaginary points $(1: \pm i: 0: 0)$.

${ }^{3}$ The geometry of turbines was initiated by Kasner [7]. An extensive bibliography on the subject is to be found in [5]. 